\title{
USE OF THE UNB_TOpoDens MODEL FOR LOCAL MODELLING OF CHOSEN GRAVITY FIELD PARAMETERS IN THE WESTERN CARPATHIANS
}

\author{
Marek TROJANOWICZ ${ }^{1)}$, Lubomil POSPÍŠIL ${ }^{2)}$ and Olgierd JAMROZ ${ }^{1)}$ \\ 1) Wroclaw University of Environmental and Life Sciences, Institute of Geodesy and Geoinformatics, \\ Grunwaldzka 53, 50-357 Wroclaw, Poland \\ 2) V̌̆B - Technical University Ostrava, 17. listopadu 2172/15, 70800 Ostrava - Poruba, Czech Republic
}

*Corresponding author's e-mail: marek.trojanowicz@upwr.edu.pl

\section{ARTICLE INFO}

\section{Article history:}

Received 14 November 2019

Accepted 30 January 2020

Available online 6 February 2020

Keywords:

Differences between geoid undulations and height anomalies

Bouguer gravity anomaly in geodesy

Height systems

\begin{abstract}
The latest, global density model of topographical masses (UNB_TopoDens model) was used to determine complete Bouguer anomalies and the differences between geoid undulations and height anomalies in an area of the Western Carpathians, within the Polish-Slovak-Czech border region. The ranges of the calculated values are between approximately -74 and $+23 \mathrm{mGal}$ for Bouguer anomalies and -10 and $+5 \mathrm{~cm}$ for differences between geoid and quasigeoid heights. Calculations were also performed for constant densities of topographical masses $\left(2200,2500\right.$ and $\left.2670 \mathrm{~kg} / \mathrm{m}^{3}\right)$. The results show significant differences due to the various densities adopted for the calculations. For example, differences between the version using densities from the UNB_TopoDens model and the version using a constant density of $2670 \mathrm{~kg} / \mathrm{m}^{3}$ ranged between $-3.4 \mathrm{mGal}$ and $+37.5 \mathrm{mGal}$, for Bouguer anomalies, and between $-0.5 \mathrm{~cm}$ and $+7.4 \mathrm{~cm}$ for differences between geoid undulation and height anomalies. It has also been shown that, for high mountain areas, differences between geoid and quasigeoid height should be determined on the basis of exact formula (not on the basis of Bouguer anomalies).
\end{abstract}

\section{INTRODUCTION}

Orthometric and normal height systems are the basis for defining national height reference systems. The main problem associated with the use of classic orthometric heights, using the geoid as the reference surface, is associated with insufficient knowledge of the topographic mass density distribution (Heiskanen and Moritz, 1967; Torge, 2001; Sjöberg, 2018). As Foroughi et al. (2017) showed, accurate, local and regional models of topographic mass density can allow the geoid model to be determined to the same level of accuracy as the quasigeoid model. Thus, it is possible to determine orthometric heights with the same accuracy as normal heights. Such models were already used in geoid modelling (e.g. Martinec and Vaníček, 1994; Huang et al., 2001) however, this was not common practice due to the lack of relevant data on a global scale. A new global topographic mass density model is now available (UNB_TopoDens, Sheng et al., 2019), which prompts us to re-examine the differences between these two basic height systems.

The main purpose of this study is to use the aforementioned topographic mass density model to conduct analyses regarding the differences between geoid undulations and height anomalies (which are also differences between normal and orthometric heights) for a selected area.

\section{THEORETICAL FOUNDATIONS OF THE ANALYSES}

The basic relationships between normal $\left(H^{N}\right)$, orthometric $\left(H^{o}\right)$ and ellipsoidal $(h)$ heights as well as relationship between geoid undulation $(N)$ and height anomaly $(\zeta)$, can be shown as in Figure 1:

$$
\begin{aligned}
& h=H^{o}+N=\frac{C}{\bar{g}}+N \\
& h=H^{N}+\zeta=\frac{C}{\bar{\gamma}}+\zeta
\end{aligned}
$$

where $C$ is the geopotential number, $\bar{g}$ is the average gravity between the geoid and point $P(3)$, and $\bar{\gamma}$ is the average normal gravity between the ellipsoid and telluroid (4).

$$
\begin{aligned}
& \bar{g}=\frac{1}{H^{o}} \int_{0}^{H^{o}} g d H^{o} \\
& \bar{\gamma}=\frac{1}{H^{N}} \int_{0}^{H^{N}} \gamma d H^{N}
\end{aligned}
$$

Determination of $\bar{g}$ based on equation (3) is impossible due to the lack of data. In practice, this value is often calculated on the basis of Poincaré and Prey reduction (Hofmann-Wellenhof and Moritz, 2005) which is supplemented here by the influence of the atmosphere. According to this procedure, the influence of topography (topographic reduction - 


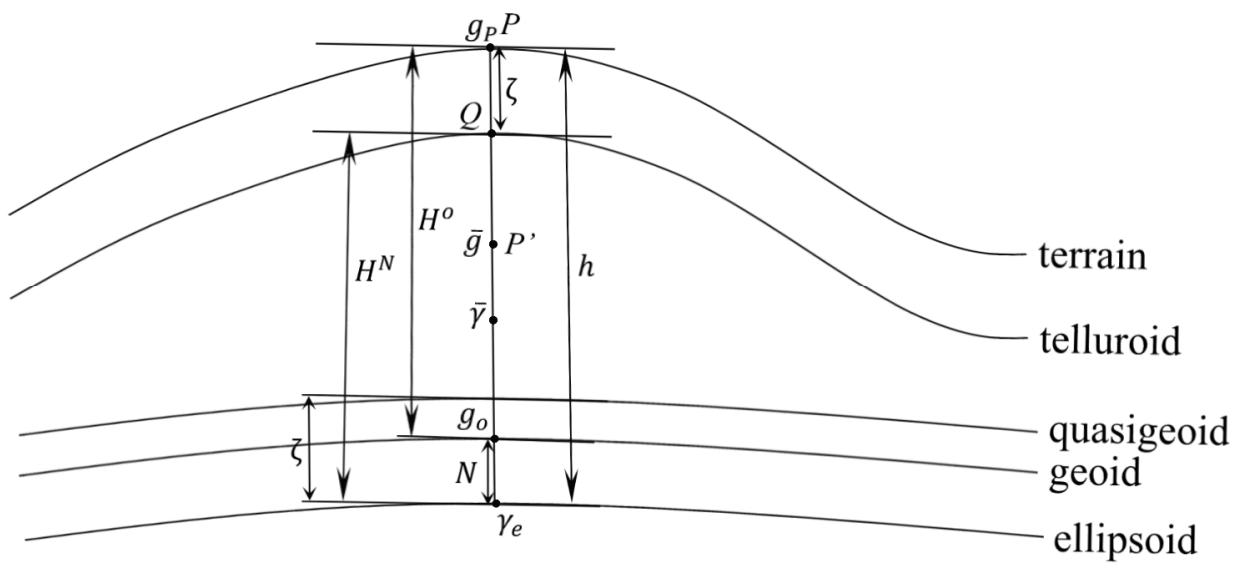

Fig. 1 Basic relationship between orthometric and normal heights. Further explanation can be found in the text.

$\left.\delta g_{\text {Top }}\right)$ and the atmosphere (atmospheric correction $\left.\delta g_{\text {atm }}\right)$ is removed from the gravity at point $P$. Freeair reduction $\left(\frac{\partial \gamma}{\partial H} \frac{H}{2}\right)$ is introduced to transfer gravity to a point $P^{\prime}$ lying at the middle height of point $P$. The topographic reduction $\left(\delta g_{\text {Top }}^{\prime}\right)$ and atmospheric correction $\left(\delta g_{a t m}^{\prime}\right)$ for point $P^{\prime}$ is determined and restored:

$$
\bar{g}=g_{P}-\frac{\partial \gamma}{\partial H} \frac{H}{2}-\delta g_{T o p}+\delta g_{T o p}^{\prime}+\delta g_{a t m}-\delta g_{a t m}^{\prime}
$$

The atmospheric correction, in less precise calculations, is used in its simplified form (Hinze et al., 2005):

$$
\delta g_{a t m}=0.874-9.9 \times 10^{-5} h+3.56 \times 10^{-9} h^{2}
$$

Note that the influence of the atmosphere in equation (5) is very small (both removing and restoring it). For the area analysed in this paper, it reaches $0.1 \mathrm{mGal}$ (calculated by equation (6)) and so, for the determination of the orthometric heights, it can be ignored. Eventually we can write:

$\bar{g}=g_{P}-\frac{\partial \gamma}{\partial H} \frac{H}{2}-\delta g_{\text {Top }}+\delta g_{\text {Top }}^{\prime}$

It should be noted that simplified Prey reduction, including only the Bouguer slab with a constant density ( $\rho=2670 \frac{\mathrm{kg}}{\mathrm{m}^{3}}$ ), leads to the formula:

$\bar{g}=g_{P}+0.0424 H$

This defines the so called Helmert heights (Hofmann-Wellenhof and Moritz, 2005), when it is used for determining the orthometric heights.

The topographic reductions used in formulas (5) and (7), determined for point $P$ (Fig. 1), are expressed as:

$\delta g_{\text {Top }}=G \iiint_{\Omega} \frac{\left(H-H_{P}\right)}{l^{3}} \rho d V_{\Omega}$

where $l$ is the distance between the attracting masses and the attracted point $P, \rho$ is density, $G$ is Newton's gravitational constant, $\Omega$ is integration volume (topographical mass) and $d V_{\Omega}$ is the volume element. Topographic reduction is often presented in the form of the sum of the Bouguer plate and terrain correction. However, due to taking into account the curvature of the Earth and the use of variable topography densities, we use equation (9) in our calculations. The terrain correction (also terrain reduction) is usually calculated on the basis of DEM with very high resolution near the gravity point, which decreases with distance. It is also worth noting that terrain correction is usually calculated to a distance of about $167 \mathrm{~km}$ (Hinze et al., 2005), only introducing corrections for further zones in more precise and large-scale geophysical studies (Mikuška et al., 2006).

When further zones are not included, calculations can be performed in the local Cartesian coordinate system, taking into account the sphericity of the Earth. The $Z$-axis of the coordinate system is directed towards the geodetic zenith at the origin point (in the middle of the study area). The $X$ and $Y$ axes lie on the plane of the horizon of that point and are directed toward the north and east, respectively. The definition of the coordinate system enables the determination of the $\Omega$ volume in the form of rectangular prisms for which the solutions of Newton's integral (9) is presented by Nagy (1966) and Nagy et al. (2000). In practice, calculations are carried out using DEM in the form of the coordinates $x, y$, $z=H$, wherein the current DEM block is moved down by the value of $\Delta z=-\left(R-\sqrt{R^{2}-d^{2}}\right)$, where $R$ is accepted as being the Earth's radius (in the calculations we adopted $R=6371 \mathrm{~km}$ ) and $d$ is the horizontal distance of the gravity point from the DEM block centre.

Equation (9) can now be written as follows:

$$
\begin{aligned}
& \quad \delta g_{\text {Top }}= \\
& \sum_{i=1}^{n}\left(\rho_{i} G \int_{z_{i 1}}^{z_{i 2}} \int_{y_{i 1}}^{y_{i 2}} \int_{x_{i 1}}^{x_{i 2}} \frac{\left(z_{i}-z_{P}\right)}{l_{i}^{3}} d x_{i} d y_{i} d z_{i}\right)= \\
& \sum_{i=1}^{n}\left(\rho_{i} K_{i}\right)
\end{aligned}
$$


where: $n$ is the number of rectangular prisms of DEM; $x_{i 1}, x_{i 2}, y_{i 1}, y_{i 2}, z_{i 1}, z_{i 2}$ are the coordinates defining the rectangular prism $i$ of DEM; $\rho_{i}$ is its constant density;

$$
l_{i}=\sqrt{\left(x_{i}-X_{P}\right)^{2}+\left(y_{i}-Y_{P}\right)^{2}+\left(z_{i}-Z_{P}\right)^{2}} ;
$$

After subtracting equation (2) from equation (1) we are left with a formula that enables determination of the difference of the geoid height and the height anomaly, which is equal to the difference between the normal and orthometric heights:

$N-\zeta=\frac{\bar{g}-\bar{\gamma}}{\bar{\gamma}} H^{o}$ 2001):

Equation (11) is widely approximated by (Torge,

$N-\zeta \approx \frac{\Delta g_{B}}{\bar{\gamma}} H$

where $\Delta g_{B}$ is the Bouguer anomaly defined as (Torge, 1989):

$\Delta g_{B}=\Delta g_{F}-\delta g_{T o p}+\delta g_{a t m}$

The free-air gravity anomaly related to the geoid $\left(\Delta g_{F}\right)$ is expressed in the form (Torge, 2001):

$\Delta g_{F}=g_{o}-\gamma_{e}$

By assuming the equality of free-air reduction of the real and normal gravity $\left(\frac{\partial g}{\partial H} H^{o}=\frac{\partial \gamma}{\partial H} H^{N}\right)$ we can also write:

$\Delta g_{F}=g_{P}-\gamma_{Q}$

It should be noted that in equations (5) and (7) we used topographic reductions $\left(\delta g_{\text {Top }}\right)$ and $\left(\delta g_{\text {Top }}^{\prime}\right)$ for masses lying between the geoid and the earth's surface. Reduction $\delta g_{\text {Top }}$ is therefore the same as in the Bouguer anomalies (13). By assuming $\bar{\gamma}=\gamma_{e}+$ $\frac{\partial \gamma}{\partial H} \frac{H}{2}$, we can write:

$$
\begin{gathered}
(\bar{g}-\bar{\gamma})=g_{P}-\frac{\partial \gamma}{\partial H} H-\delta g_{T o p}+\delta g_{\text {Top }}^{\prime}+\delta g_{a t m}- \\
\delta g_{a t m}^{\prime}-\gamma_{e}
\end{gathered}
$$
(16):

And subtracting equation (13) from equation

$(\bar{g}-\bar{\gamma})-\Delta g_{B}=\delta g_{\text {Top }}^{\prime}-\delta g_{a t m}^{\prime}$

Hence, the approximation (12) is accurate for flat areas, where the masses above and below point $P$ ' balance each other $\left(\delta g_{\text {Top }}^{\prime} \approx 0\right)$. There will be mass imbalances in mountainous areas. The atmospheric correction $\delta g_{\text {atm }}^{\prime}$ is small and reaches $0.87 \mathrm{mGal}$ for the area analysed in the paper (value calculated by (6)).

\section{DATA AND ANALYSIS OF THE RESULTS.}

The area of study included part of the Western Carpathians that lie on the border of Poland, Slovakia and the Czech Republic. In the calculations two versions of DEM resolution were used: $0.1 \times 0.1 \mathrm{~km}$ (up to $10 \mathrm{~km}$ around the gravity point) and $0.5 \times 0.5 \mathrm{~km}$ (from 10 to $200 \mathrm{~km}$ around the gravity point). DEM grids were determined based on the SRTM model with a resolution of $3^{\prime \prime}$ (Fig. 2). As mentioned in the introduction, the UNB_TopoDens model (Sheng et al., 2019) was used in the calculations. It is worth mentioning that this model was developed as two versions: $\mathrm{T}$ and $\mathrm{C}$. We used the T model in our work. The Bouguer anomalies (13) and differences (11) were determined for the topographic mass density distribution taken from the UNB_TopoDens T model $\left(\rho_{U N B}\right)$ and compared with results for constant density values, marked as $\rho_{2200}=$ $2200 \mathrm{~kg} / \mathrm{m}^{3} ; \quad \rho_{2500}=2500 \mathrm{~kg} / \mathrm{m}^{3}$ and $\rho_{2670}=$ $2670 \mathrm{~kg} / \mathrm{m}^{3}$ accordingly.

In the calculations, we used a set of 67,074 points with measured gravity evenly distributed across an elaborate area (marked by a solid red line in Figure 2). Gravity points in the Polish region were made available by The Polish Geological Institute - National Research Institute (PGI-NRI) and by Geofyzika, a.s. Brno, ČGS - Geofond for the gravity points in the Czech and Slovak regions. In addition, the lack of gravity points in a small gap in the Polish part of the Tatra Mountains, was supplemented by 208 gravity values calculated from the GGI model (Trojanowicz et al., 2020). The approximate density of the gravity data was approximately 1 point per $1.2 \mathrm{~km}^{2}$.

At first, the Bouguer anomalies $\left(\Delta g_{B_{U N B}}\right)$ were calculated based on equation (13) and values of $(N-\zeta)_{U N B}$ based on equation (11) for all 67,282 points, using the density $\rho_{U N B}$. Relief maps of both quantities are presented in Figures 3 and 4.

The basic statistics of the determined values are presented in Table 1.

To analyse the significance of the UNB_TopoDens model in determining both the Bouguer anomalies and $N-\zeta$ values, calculations were carried out again for the constant densities $\rho_{2200}$, $\rho_{2500}$ and $\rho_{2670}$. The corresponding differences were then calculated:

Table 1 Basic statistics of values $\Delta g_{B}{ }_{U N B}$ and $(N-\zeta)_{U N B}$.

\begin{tabular}{lcccc}
\hline & $\max$ & $\min$ & average & stdev \\
\hline$\Delta g_{B_{U N B}}[\mathrm{mGal}]$ & 23.06 & -74.2 & -20.38 & 20.36 \\
$(N-\zeta)_{U N B}[\mathrm{~cm}]$ & 4.5 & -10.1 & -1.19 & 1.40 \\
\hline
\end{tabular}




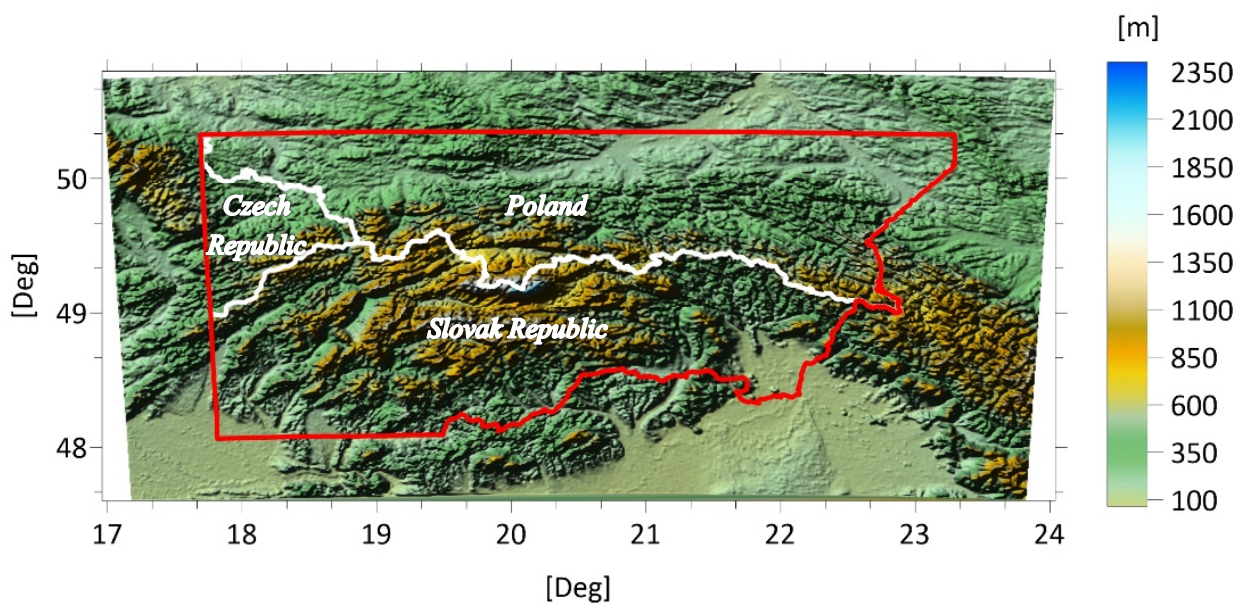

Fig. 2 Map of the topography. The solid red line represents the border of the study area and the white line, the border between Poland and the Czech and Slovak Republics.

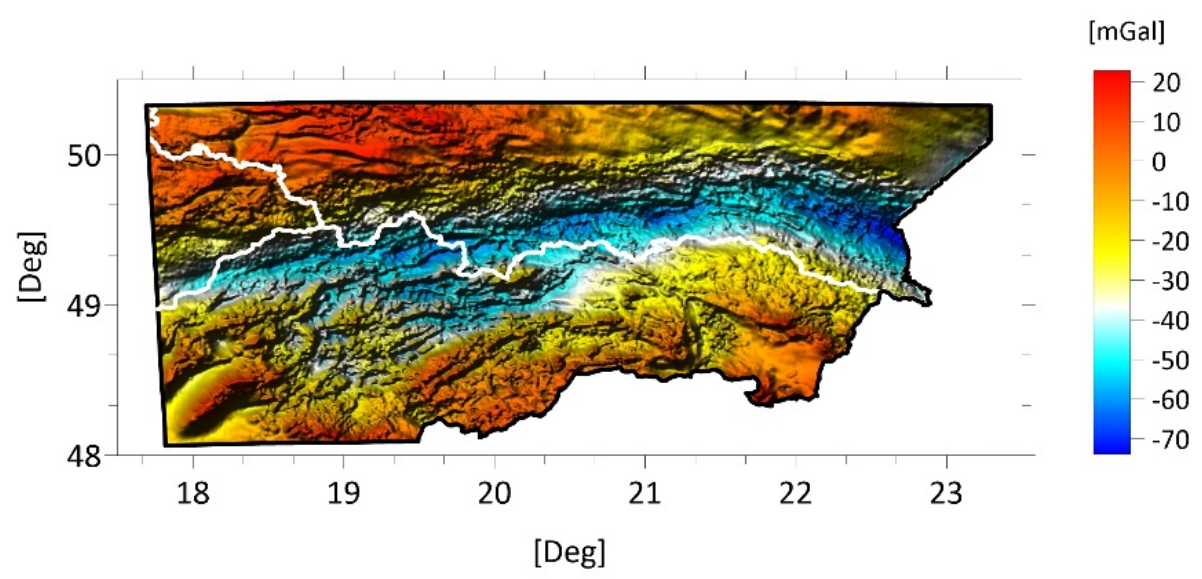

Fig. 3 Map of the complete Bouguer anomalies $\Delta g_{B U N B}$.

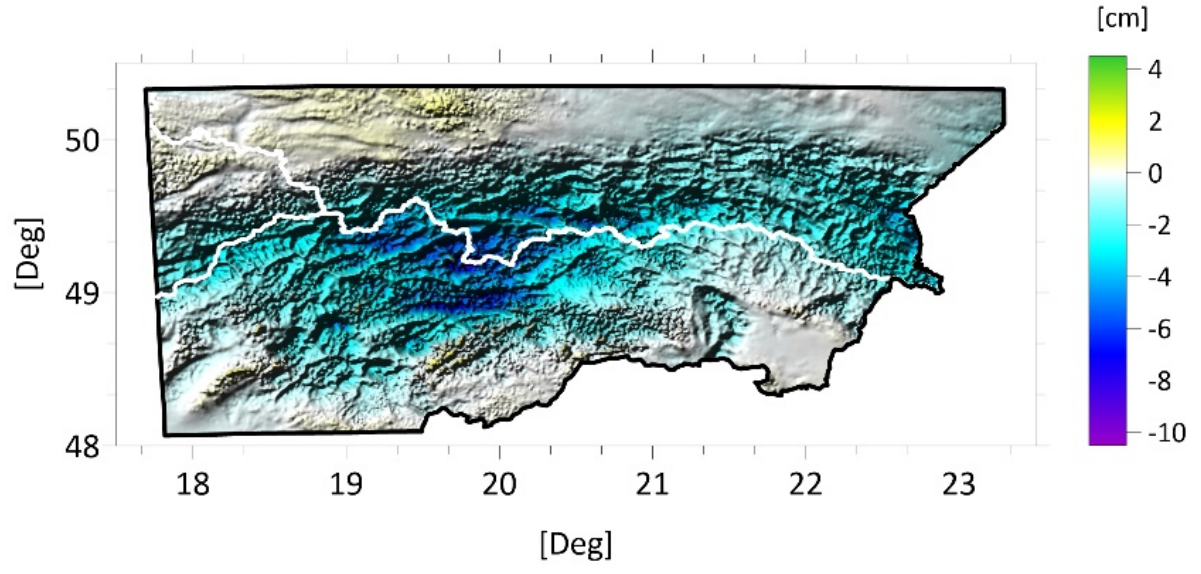

Fig. 4 Map of the differences $(N-\zeta)_{U N B}$. 
Table 2 Basic statistics of differences $\delta \Delta g_{B}$ and $\delta(N-\zeta)_{\rho}$ for three constant densities.

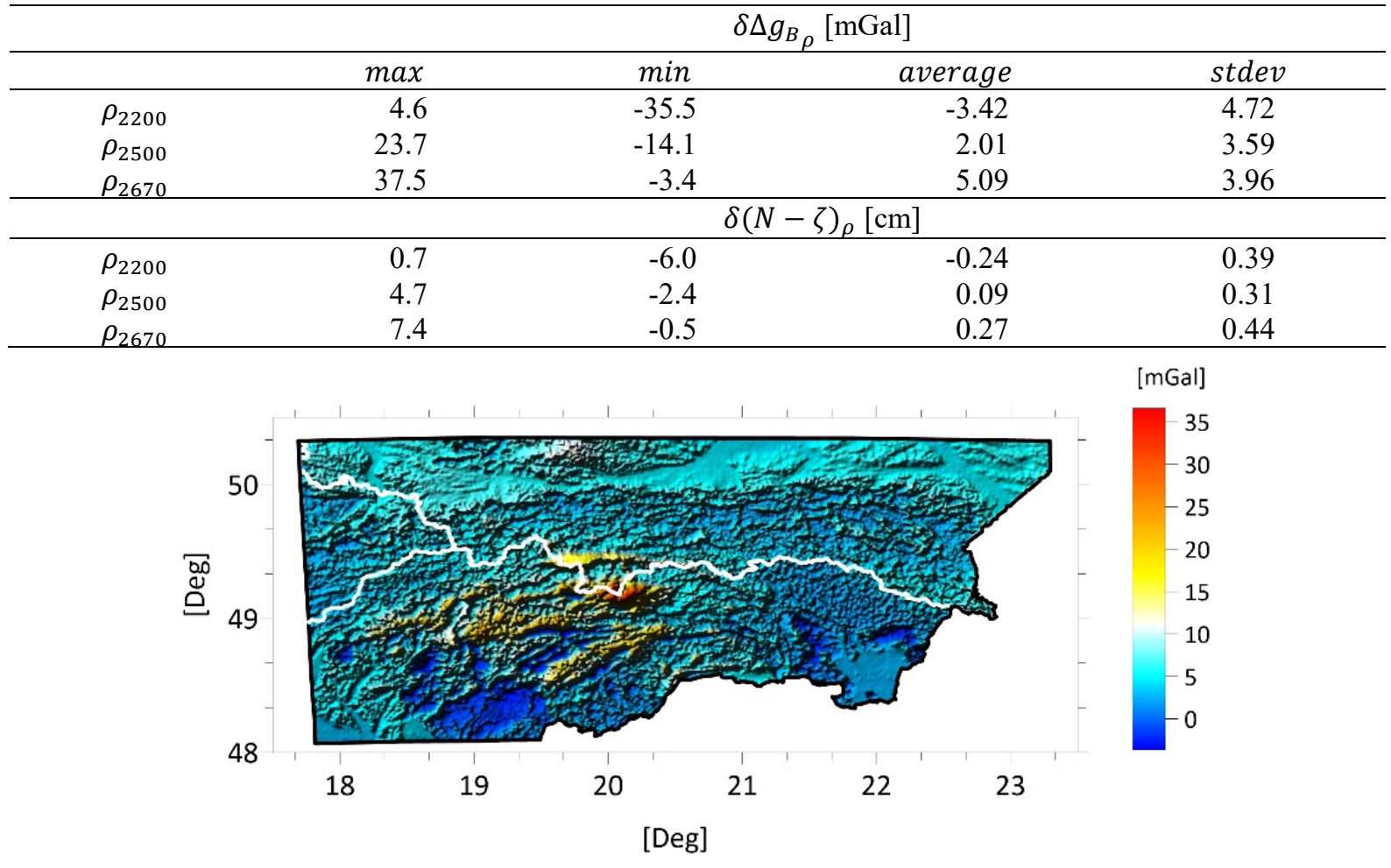

Fig. 5 Map of the values $\delta \Delta g_{B}$ for $\rho_{2670}$.

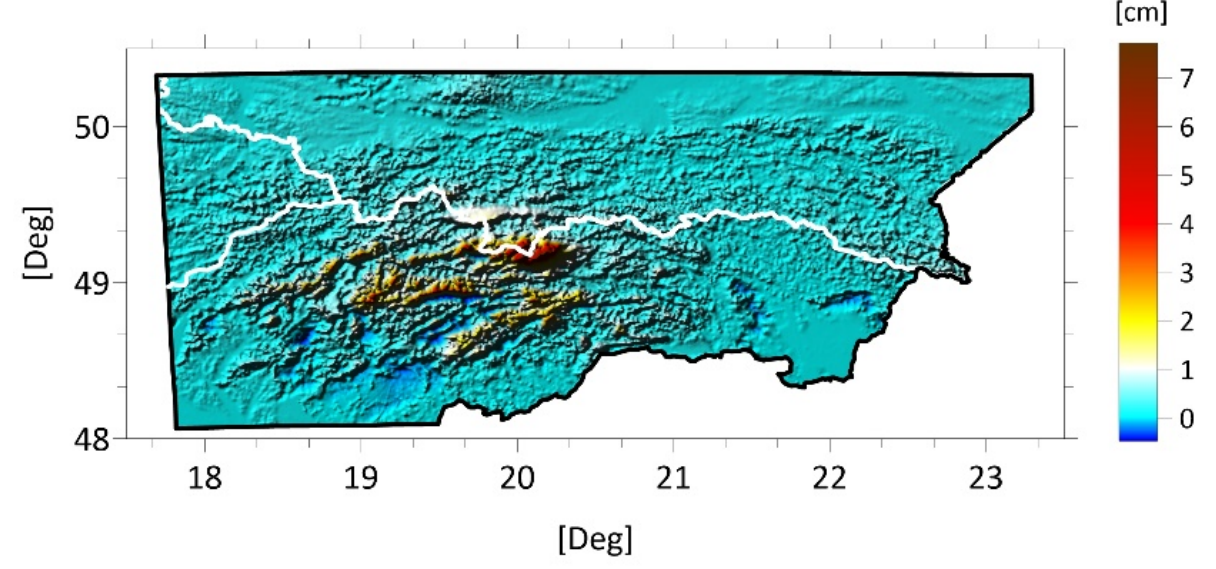

Fig. 6 Map of the values $\delta(N-\zeta)_{\rho}$ for $\rho_{2670}$.

$$
\begin{aligned}
& \delta \Delta g_{B}=\Delta g_{B}{ }_{U N B}-\Delta g_{B} \rho \\
& \delta(N-\zeta)_{\rho}=(N-\zeta)_{U N B}-(N-\zeta)_{\rho}
\end{aligned}
$$

Basic statistics for the differences (18) and (19) are presented in Table 2 .

As can be seen from the statistics presented in Table 2, different values of constant density affect both the Bouguer anomalies and $N-\zeta$ values. The range of the changes in these values and the standard deviations calculated for different densities are similar, although the most favourable results were obtained for version $\rho_{2500}$. A change in the density essentially shifts the entire range of differences by a certain amount.

Examples of the spatial distribution of the differences $\delta \Delta g_{B}$ and $\delta(N-\zeta)_{\rho}$ determined for density $\rho_{2670}$ are presented in Figures 5 and 6 , where it can clearly be seen that the largest differences for both analysed quantities occur in the highest parts of the mountains.

Supplementary to the analysis above is an estimation of the errors resulting from the 


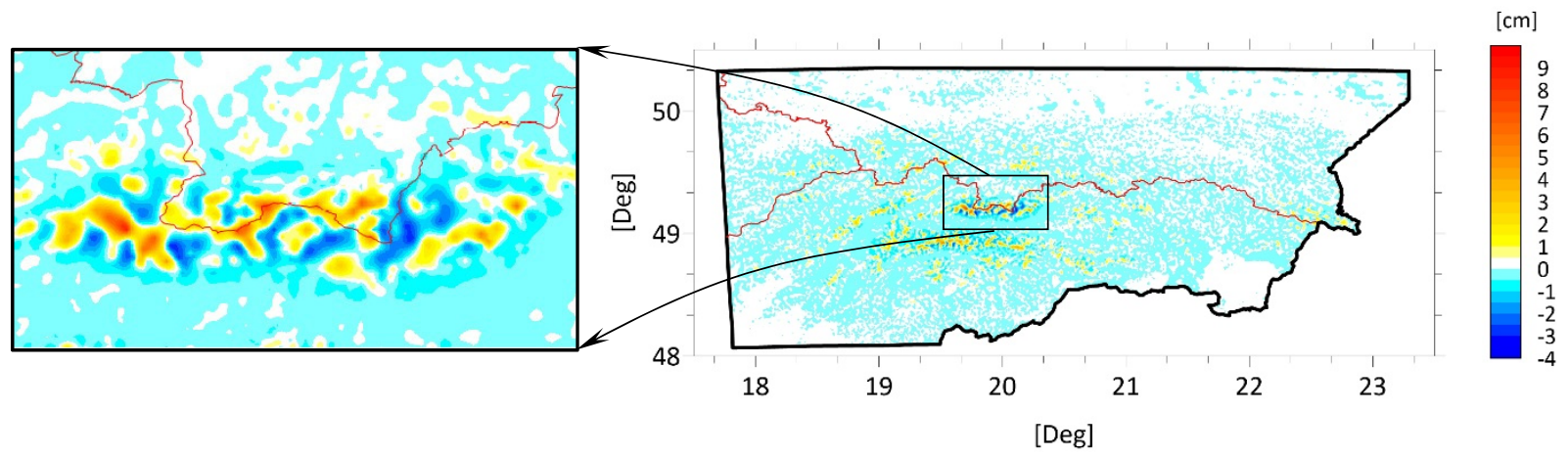

Fig. 7 Map of the values $\delta_{N-\zeta}$.

approximation (12). For this purpose, the values ( $N-$ $\zeta$ ) were determined on the basis of Bouguer anomalies and the differences were calculated:

$\delta_{N-\zeta}=\frac{\bar{g}-\bar{\gamma}}{\bar{\gamma}} H-\frac{\Delta g_{B}}{\bar{\gamma}} H$

Figure 7 shows the spatial distribution of the differences (20). Their maximum value is $9.5 \mathrm{~cm}$, minimum value is $-3.9 \mathrm{~cm}$, the average is 0.0 and standard deviation is $0.40 \mathrm{~cm}$.

Following on from the above results, the approximation given by (12) is very good and completely sufficient for most of the study area. However, in the area of the highest mountains (e.g. the Tatra Mountains), errors caused by such approximations are significant and exceed $9 \mathrm{~cm}$.

\section{CONCLUSIONS}

In this paper, complete Bouguer anomalies and differences $(N-\zeta)$ were determined using the UNB_TopoDens $T$ model for an area of the Western Carpathians. The ranges of the calculated values are approximately between -74 and $+23 \mathrm{mGal}$ for Bouguer anomalies and -10 and $+5 \mathrm{~cm}$ for differences between geoid and quasigeoid heights, which are equal to the differences between normal and orthometric heights. It was shown that the adoption of constant densities for the topographic masses in the calculations will significantly affect the determined complete Bouguer anomalies and $(N-\zeta)$ differences. The range of changes compared to the versions using the UNB_TopoDens model, reached almost $40 \mathrm{mGal}$ for Bouguer anomalies and $8 \mathrm{~cm}$ for $(N-\zeta)$ differences. Moreover, the $(N-\zeta)$ differences for mountainous areas should be determined only from the exact formula given in equation (11). The use of complete Bouguer anomalies to calculate these differences (equation (12)) in the highest parts of the analysed area changed their values by up to $9 \mathrm{~cm}$.

\section{REFERENCES}

Foroughi, I., Vaníček, P., Sheng, M., Kingdon, R.W. and Santos, M.C.: 2017, In defense of the classical height system. Geophys. J. Int., 211, 2, 1154-1161. DOI: $10.1093 /$ gji $/$ ggx366
Heiskanen, W.A. and Moritz, H.: 1967, Physical Geodesy. San Francisco W. H. Freeman and Company, San Francisco and London.

Hinze, W.J., Aiken, C., Brozena, J., Coakley, B., Dater, D., Flanagan, G., Forsberg, R., Hildenbrand, T., Keller, G.R., Kellogg, J., Kucks, R., Li, X., Mainville, A., Morin, R., Pilkington, M., Plouff, D., Ravat, D., Roman, D., Urrutia-Fucugauchi, J., Véronneau, M., Webring, M. and Winester, D.: 2005, New standards for reducing gravity data: The North American gravity database. Geophysics, 70, J25-J32.

DOI: $10.1190 / 1.1988183$

Hofmann-Wellenhof, B. and Moritz, H.: 2005, Physical Geodesy. Springer-Verlag Wien.

Huang, J., Vaníček, P., Pagiatakis, S.D. and Brink, W.: 2001, Effect of topographical density on the geoid in the Rocky Mountain. J. Geod., 74, 11-12, 805-815.

Martinec, Z. and Vaníček, P.: 1994, Direct topographical effect of Helmert's condensation for a spherical approximation of the geoid. Manuscr. Geodaet., 19, 257-268.

Mikuška, J., Pašteka, R. and Marušiak, I.: 2006, Estimation of distant relief effect in gravimetry. Geophysics, 71, 6, J59-J69. DOI: 110.1190/1.2338333

Nagy, D.: 1966, The gravitational attraction of right angular prism. Geophysics, 31, 2, 362-371.

Nagy, D., Papp, G and Benedek, J.: 2000, The gravitational potential and its derivatives for the prism. J. Geod., 74, 7, 552-560. DOI: 10.1007/s001900000116

Sheng, M.B., Vaníček, P., Kingdon, R.W., Santos, M. and Foroughi, I.: 2019, Formulation and validation of a global laterally varying topographical density model. Tectonophysics, 762, 45-60. DOI: $10.1016 /$ j.tecto.2019.04.005

Sjöberg, L.E.: 2018, On the topographic bias and density distribution in modelling the geoid and orthometric heights. J. Geod. Sci., 8, 1, 30-33.

DOI: $10.1515 /$ jogs-2018-0004

Torge, W.: 1989, Gravimetry. Walter de Gruyter, Berlin New York.

Torge, W.: 2001, Geodesy, Third edition. Walter de Gruyter, Berlin New York.

Trojanowicz, M., Pospíšil, L. and Jamroz, O.: 2020, The GGI model as a multifunctional local model of a gravity potential - case study for the Western Carpathians area. Submitted to Scientific Reports. 